\title{
Molecular Models of Amino Acids, Peptides, and Proteins
}

Cite as: Review of Scientific Instruments 24, 621 (1953); https://doi.org/10.1063/1.1770803

Submitted: 25 August 1952 . Published Online: 29 December 2004

Robert B. Corey, and Linus Pauling

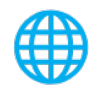

\section{ARTICLES YOU MAY BE INTERESTED IN}

Density-functional thermochemistry. III. The role of exact exchange

The Journal of Chemical Physics 98, 5648 (1993); https://doi.org/10.1063/1.464913

A consistent and accurate ab initio parametrization of density functional dispersion correction (DFT-D) for the 94 elements H-Pu

The Journal of Chemical Physics 132, 154104 (2010); https://

doi.org/10.1063/1.3382344

\section{Atomic Screening Constants from SCF Functions}

The Journal of Chemical Physics 38, 2686 (1963); https://doi.org/10.1063/1.1733573

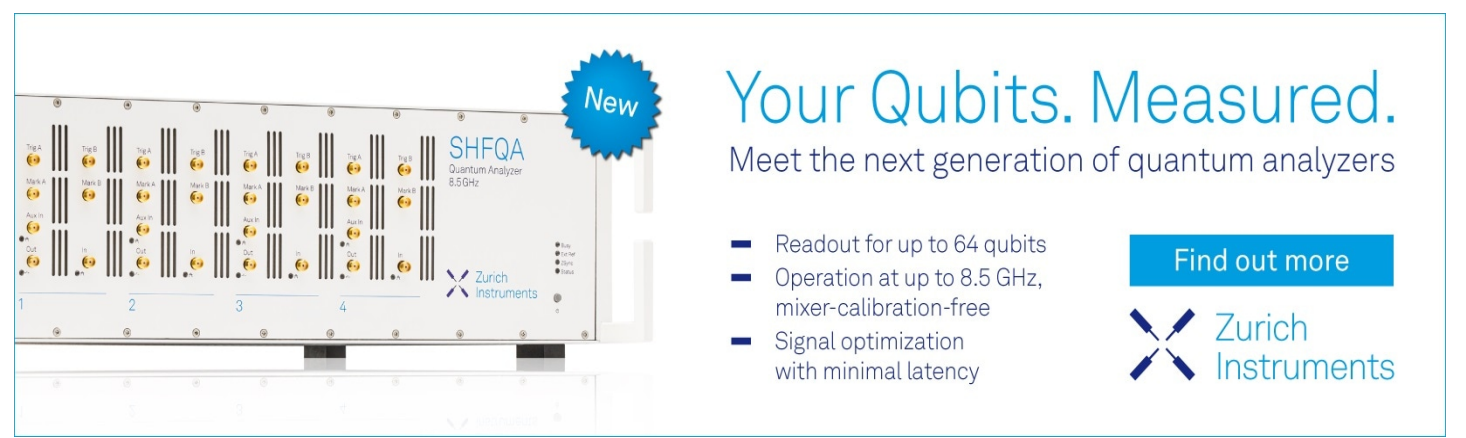




\title{
Molecular Models of Amino Acids, Peptides, and Proteins
}

\author{
Robert B. Corey and Linus Pauling \\ Gates and Crellin Laboratories of Chemistry, ${ }^{*}$ California Institule of Technology, Pasadena, California $\dagger$
}

(Received August 25, 1952)

\begin{abstract}
A set of accurate scale models has been developed for use in studies of the structures of amino acids, peptides, and proteins. Models representing atoms or groups of atoms built from hard wood to the scale 1 in $=1 \mathrm{~A}$ are connected by a clamping device which maintains desired molecular configurations. These accurate models have been used as substitutes for calculation in investigations of the probable configuration of the polypeptide chain in proteins. Analogous models constructed of rubber-like plastic to the scale $1 \mathrm{in} .=2 \mathrm{~A}$ and connected by snap fasteners are designed for qualitative studies of protein structure.
\end{abstract}

\section{INTRODUCTION}

$\mathrm{F}^{\circ}$ OR several years we have been working at the California Institute of Technology on the development of scale models for representing the molecules of amino acids and related compounds as an aid in our attack on the problems of the structure of proteins. The development of these models has closely paralleled our x-ray diffraction studies of amino acids, peptides, and proteins. Our first models were constructed in accordance with the dimensions experimentally obtained from complete and accurate determinations of the structures of amino

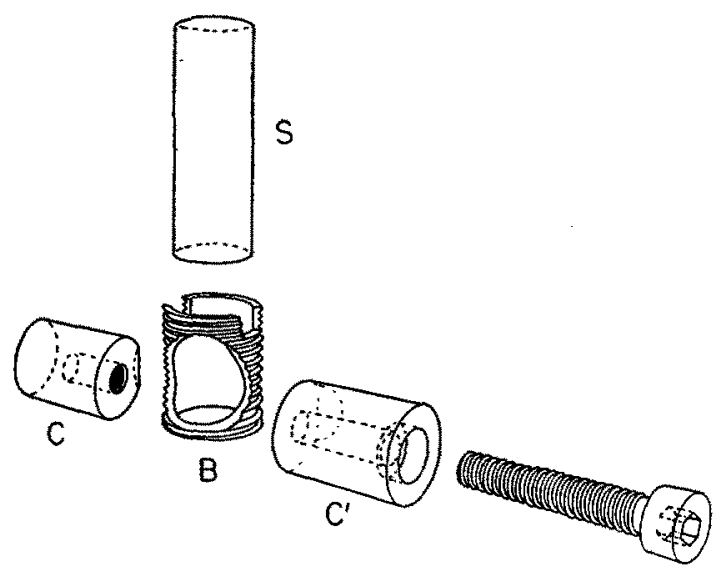

FrG. 1. An exploded view of the device used for clamping the atom models rigidly to the connecting cylindrical shafts. The threaded bushing $B$ imbedded in the atom (see Fig. 2) is intersected on one side by a hole drilled in the atom and containing the two clamping $\left(C, C^{\prime}\right)$ jaws. The latter are cut away so as to grip firmly the $\frac{5}{16}$-in. steel shaft $S$ (see Fig. 3 and following figures).

acids. They were progressively revised and extended as more accurate data from peptides and additional amino acids became available. Recently we have been engaged in a study of the structure of proteins which has led us to propose several new configurations of the polypeptide chain. Because these proposed configurations are explicitly described in terms of atomic coordinates, their occurrence in proteins can be tested by com-

* Contribution No. 1731.

$\uparrow$ Aided by a grant from the National Foundation for Infantile Paralysis. parison of their theoretically calculated $x$-ray diffraction patterns with those derived experimentally from the proteins themselves. ${ }^{1}$ In this study our molecular models have been of great assistance, especially in the recognition of sterically probable configurations of the polypeptide chain and the rejection of sterically improbable ones. ${ }^{2}$

With the continued refinement of $x$-ray diffraction measurements and the accumulation of new structural data, future revision of some dimensions will doubtless be desirable and models of additional atoms and radicals will be designed and constructed. Nevertheless the demonstrated usefulness of these models in their present form seems to justify a description of them at this time.

\section{REQUIREMENTS AND SPECIFICATIONS}

In models designed for studying probable molecular configuration and intermolecular packing the van der
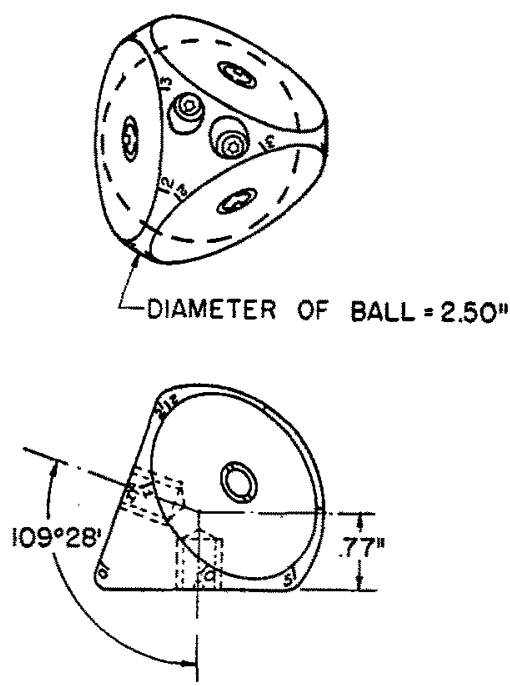

Fro. 2. A drawing of the tetrahedral carbon atom. The positions of two of the four clamps and of fiducial marks are indicated. Each face is 0.77 in. from center of ball. The ends of the steel bushings are shown in each face.

${ }_{1}$ Pauling, Corey, and Branson, Proc. Natl. Acad. Sci. U. S. 37, 205 (1951). L. Pauling and R. B. Corey, Proc. Natl. Acad. Sci. U. S. 37, 235, 241, 251,256, 261, 272, 282 (1951).

${ }^{2}$ L. Pauling and R. B. Corey, Proc. Natl. Acad. Sci. U. S. 37, 729 (1951); Proc. Natl. Acad. Sci. U. S. 38, 86 (1952). 
Waals radii of the atoms should conform to the intermolecular distances found in crystals and in noncrystalline solids rather than to gas collison radii or even smaller radii commonly used in models of organic molecules. Intramolecular interferences between atoms attached to the same atom or group of atoms must be avoided unless there is experimental or theoretical justification for believing that the interferences occur. If the models are intended for use as a substitute for

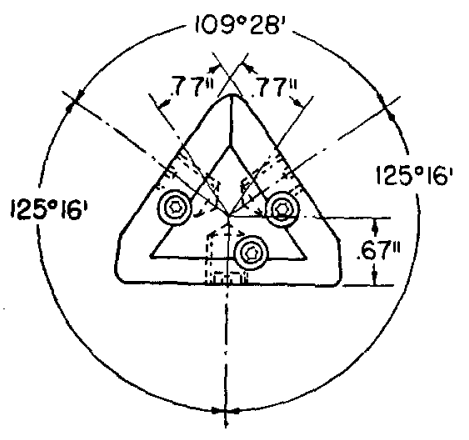

Fig. 3(a). A drawing of the double-bonded carbon atom. In the interest of clarity, only one clamp is shown in the view along the double-bond direction.
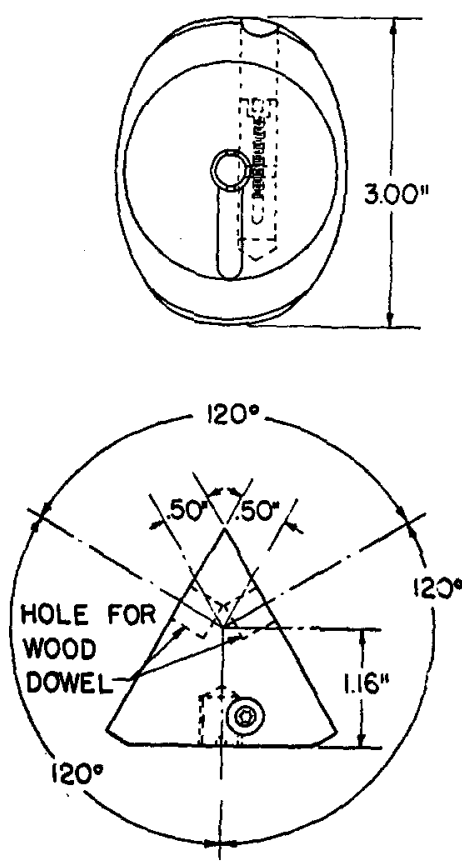

Fic. 3(b). A drawing of the aromatic carbon atom. Six atoms are fastened together permanently to form the benzene nucleus. calculation in the examination of structures, they must be accurately built and capable of retaining their bond angles and other configurational features without deformation; the scale should be conveniently large to permit easy handling and to allow angles and distances to be measured with satisfactory precision. In order to meet even these minimum requirements, many compromises were found to be necessary.

The models are made of hard wood to the scale, $1 \mathrm{in.}$ $=1 \mathrm{~A}$. Van der Waals radii, bond radii, and bond angles have the dimensions generally found in crystals of amino acids, peptides and other organic compounds. Atoms or assemblies of atoms, as the benzene nucleus and amide group, are joined together by means of short
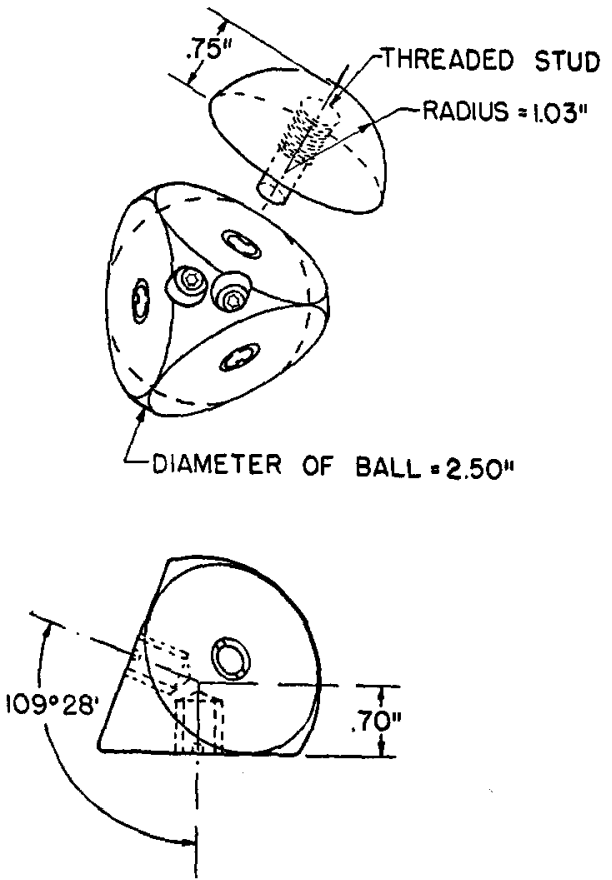

Fic. 4. A drawing of the tetrahedral positively charged nitrogen atom. For representing the neutral atom, the adapter applies an appropriate van der Waals radius to one of the bond positions.

pieces of $\frac{5}{16}$-in. steel rod which fit into steel bushings imbedded in the atoms. For rigidly fixing the relative orientation of atoms around a bond, the bushings are locked on the rod in any desired position by means of clamping fixtures also built into the atom. An exploded assembly drawing of the steel stud, bushing, and clamping device used to connect the atoms is shown in Fig. 1. Models of carbon, nitrogen, oxygen, and hydrogen atoms, and of typical assemblies are described in the following paragraphs.

\section{The Carbon Atom}

A drawing of the tetrahedral carbon atom is shown in Fig. 2. The bond radius is 0.77 in. Since the tetrahedral carbon atom is entirely surrounded by bonded atoms and so in general makes no van der Waals con- 
(a)
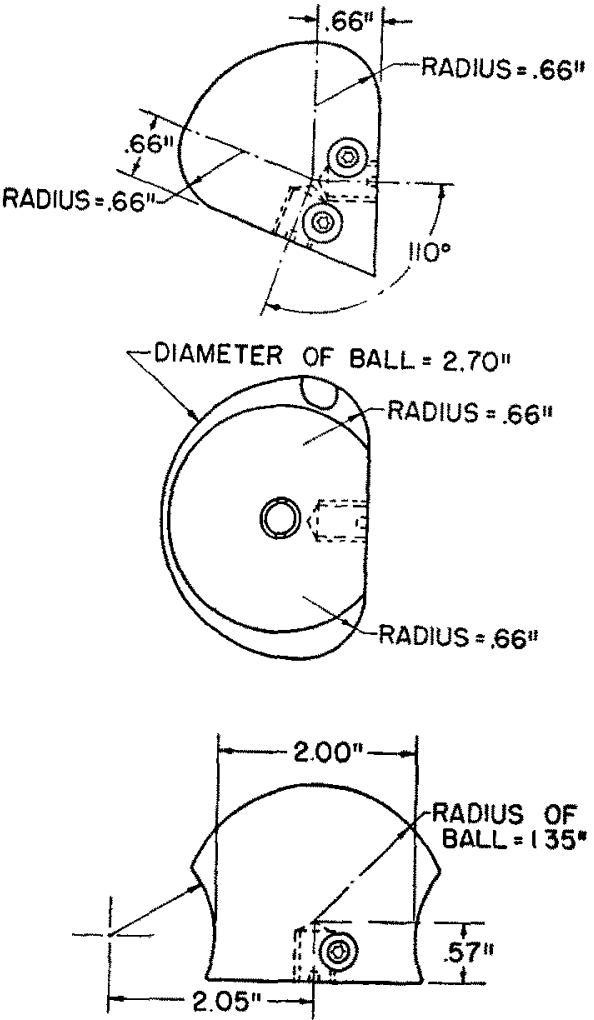

(b)

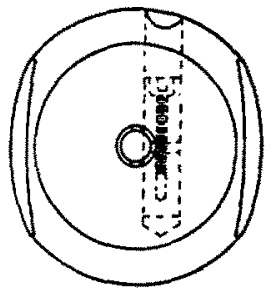

Frg. 5. Drawings of the (a) single-bonded and (b) double-bonded oxygen atom.

tacts, the radius of the ball (1.25 in.) bears no relationship to the van der Waals radius $(1.5 \mathrm{~A})$ of carbon, but was selected for reasons of convenience. When this atom is incorporated in the polypeptide chain, fiduciary marks (numbered $0,1,2,3,4,5$ ) $60^{\circ}$ apart around the edges of two faces register with marks on the amide group for indicating the orientation around the single bond.

A double-bonded carbon atom is shown in Fig. 3a. The single and double bond radii are 0.77 and $0.67 \mathrm{in}$., respectively. In the nonbonded direction the radius of the ball (1.50 in.) corresponds to the van der Waals radius of carbon. The slot on the double-bond face receives a key to determine the orientation and prevent rotation around the double bond.

Aromatic carbon atoms (Fig. 3b) are built into permanent six-membered benzene nuclei. The single bond

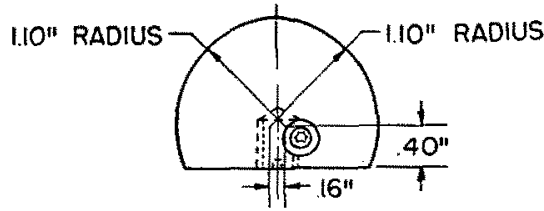

Frg. 6. A drawing of the hydrogen atom.

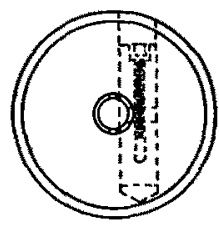

radius and the carbon-carbon distance within the ring are $0.77 \mathrm{in.}$ and $1.39 \mathrm{in}$., respectively.

\section{The Nitrogen Atom}

The positively charged tetrahedral nitrogen atom is shown in Fig. 4. It can be transformed into the neutral atom by the use of an adapter which applies an appropriate van der Waals radius to one of the four bond positions. The amide nitrogen atom is described below as part of the amide group.

\section{The Oxygen Atom}

The dimensions of the single- and double-bonded oxygen atoms are shown in Fig. 5. The latter is relieved
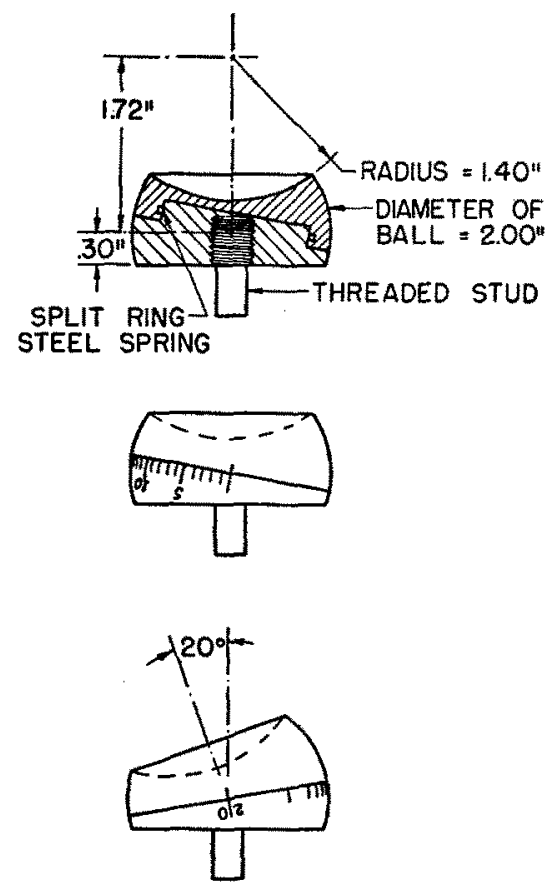

Fig. 7. A drawing of the hydrogen-bonding hydrogen atom showing how the $\mathrm{H} \cdots \mathrm{O}$ vector may be inclined at angles of from $0^{\circ}$ to $20^{\circ}$ to the $\mathrm{N}-\mathrm{H}$ bond direction by rotation of one section of the model relative to the other. 

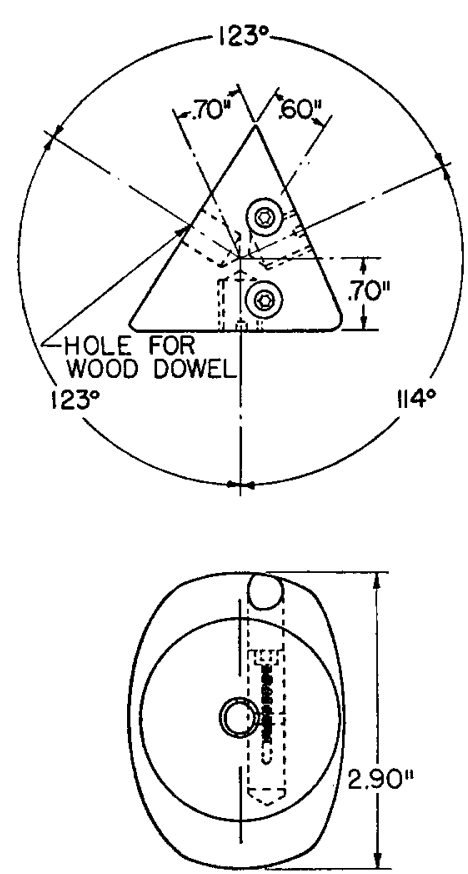

(a)
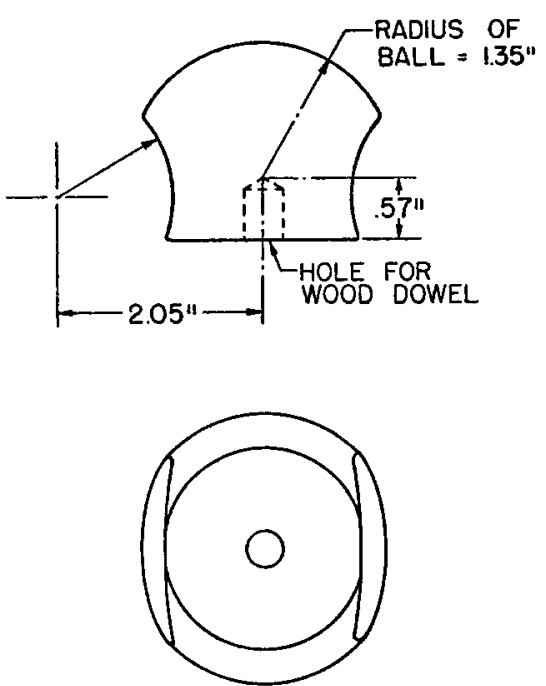

(c)
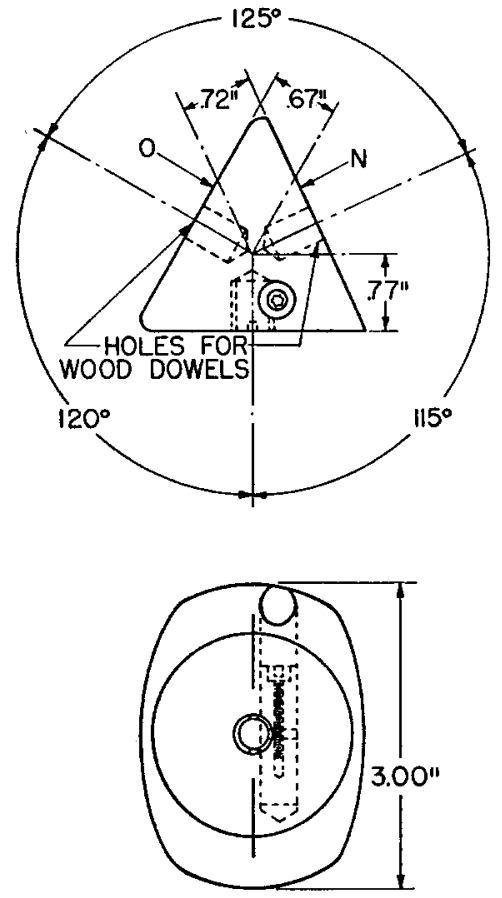

(b)
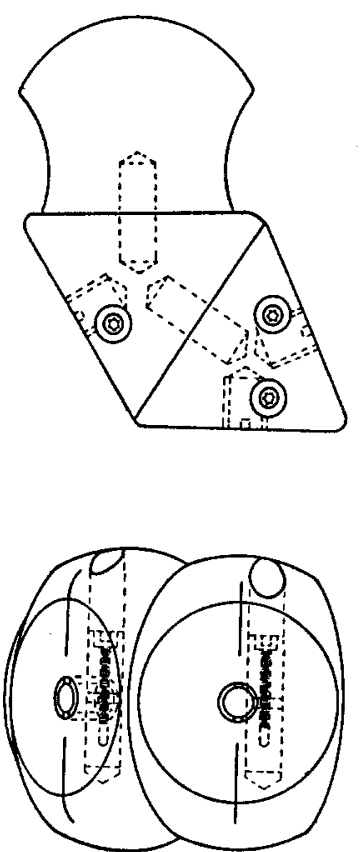

(d) on opposite sides to permit the near intramolecular approach of atoms bonded to the atom to which the oxygen is attached.

\section{The Hydrogen Atom}

A drawing of a model of the hydrogen atom representing a bond radius of $0.30 \mathrm{~A}$ and a van der Waals radius of $1.20 \mathrm{~A}$ are shown in Fig. 6 . At large angles to the direction of the bond, the van der Waals radius is
Fic. 8. A drawing of the amide group showing the dimensions of the (a) nitrogen, (b) carbon, and (c) oxygen atoms and (d) the assembled group. The three atoms are glued together to form a rigid unit. somewhat reduced to permit closer intramolecular approach of atoms.

The formation of $\mathrm{N}-\mathrm{H} \cdots \mathrm{O}$ hydrogen bonds is an important factor governing the arrangement of the molecule in crystals of amino acids and simple peptides. In proteins the configuration of the molecules and their specific spatial arrangements in crystals and fibers is directly related to their peculiar capacities for hydrogen bond formation. Our crystal structure studies of amino 


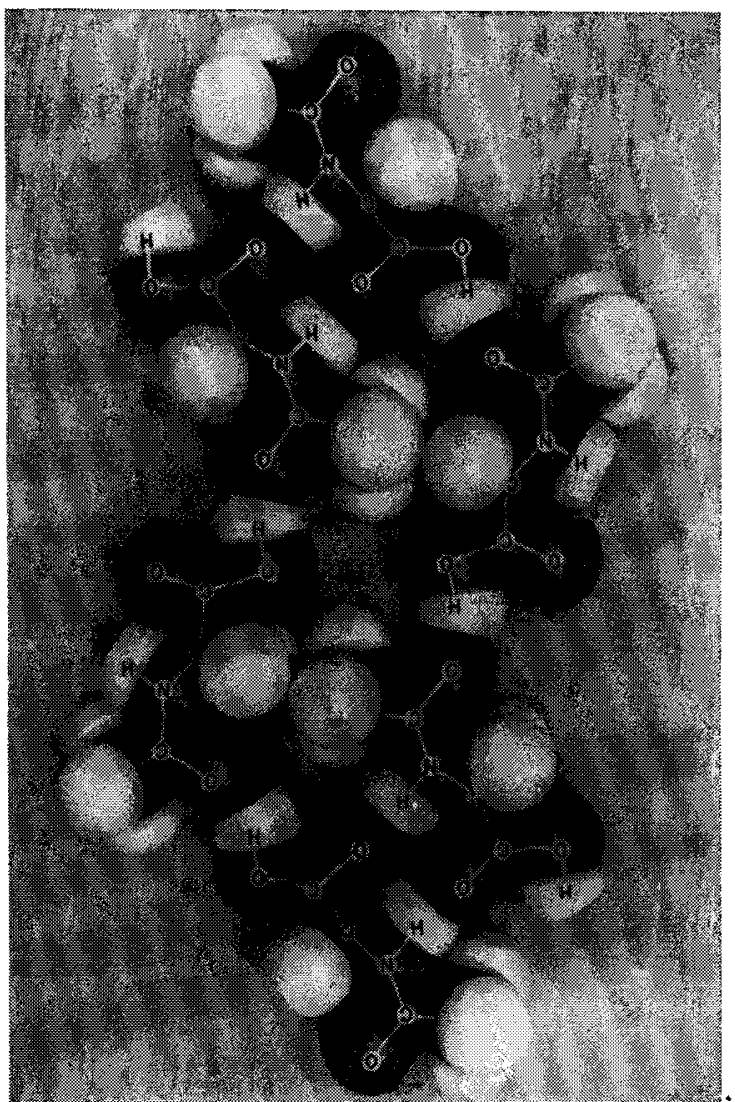

Fig. 9. A photograph of a scale model of one molecular layer in a crystal of $\mathrm{N}$-acetylglycine, showing how the molecules are held together by $\mathrm{O}-\mathrm{H} \cdots \mathrm{O}$ and $\mathrm{N}-\mathrm{H} \cdots \mathrm{O}$ hydrogen bonds.

acids and peptides have shown that in $\mathrm{N}-\mathrm{H} \cdots \mathrm{O}$ bonds the distance between the nitrogen and oxygen atoms generally falls within the range $2.79 \pm 0.12 \mathrm{~A}$ and the $\mathrm{H} \cdots \mathrm{O}$ vector usually makes an angle of less than $20^{\circ}$ with the probable direction of the $\mathrm{N}-\mathrm{H}$ bond. These dimensions have been incorporated in a model of the hydrogen-bonding hydrogen atom illustrated in Fig. 7. The model is composed of two sections held together by a spring snap ring. When a model of an oxygen atom rests in the depression its center is approximately $2.70 \mathrm{in}$. from the center of the nitrogen atom to which the hydrogen atom is attached. Rotation of the two parts of the hydrogen atom relative to one another inclines the $\mathrm{H} \cdots \mathrm{O}$ vector from $0^{\circ}$ to $20^{\circ}$ with respect to the direction of the $\mathrm{N}-\mathrm{H}$ bond. The angle of inclination can be read on a scale.

\section{The Amide Group}

The polypeptide chain<smiles>[R]C([R])(C)N([2H])C(=O)C([R2])([R2])N([2H])C(=O)C([R3])([R3])N(C)C(C)=O</smiles>

may be regarded as a series of tetrahedral carbon atoms (to each of which are attached a hydrogen atom and a side chain $R$ ) 'connected by amide groups. The amide

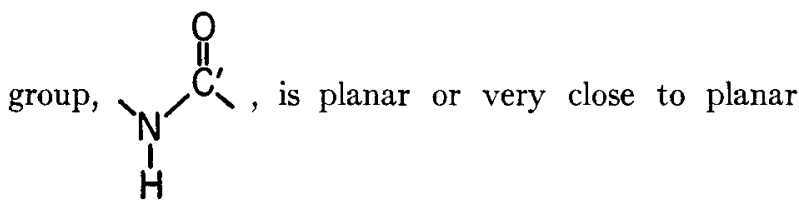

because of the resonance of the double bond between the carbon-oxygen and the carbon-nitrogen positions. A model of the amide group is shown in Fig. 8. The bond lengths represented are doubtless close to those which occur in peptides and proteins. The bond angles around the nitrogen and the carbon atoms are probably not so accurate, both because experimental data are still insufficient to establish them with certainty and because compromises have been made to increase the simplicity and to extend the usefulness of the models. It is unlikely that any of these angles differs by greater than $3^{\circ}$ from their average value in proteins.

\section{The Side Chains}

Many of the side chains of proteins can be constructed from the models for carbon, hydrogen, oxygen, and

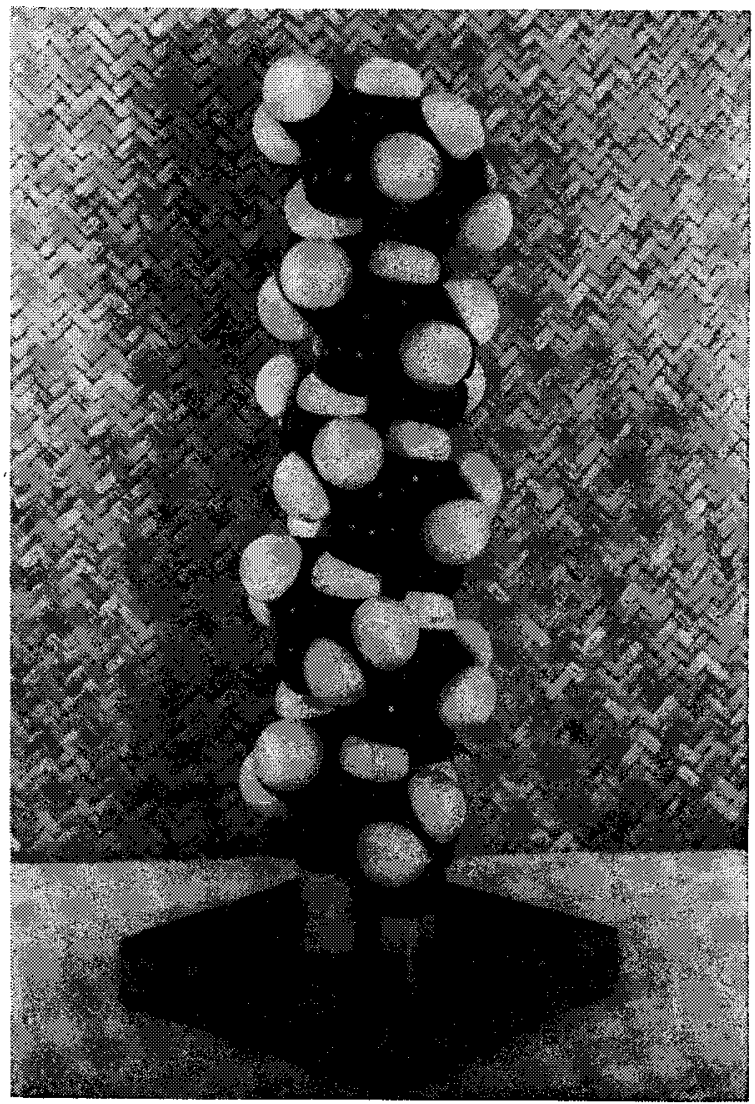

FIG. 10. A photograph of a model of the polypeptide chain in the configuration of the $\alpha$ helix, illustrating hydrogen bonding between adjacent turns of the helix. 
nitrogen atoms already described. The benzene nucleus of phenylalanine and tyrosine is made up as a single permanent unit rather than assembled from separate pieces representing the aromatic carbon atom. Because of the rigidity and close tolerances of the bond angles, it is necessary to use a slight variant of the tetrahedral carbon atom for the construction of a model of the five membered ring of proline representing the configuration found in crystals. ${ }^{3}$ We have not yet built models of the special groups in histidine, arginine, and tryptophan, the dimensions of which have yet to be established by $\mathrm{x}$-ray analysis.

\section{APPLICATIONS OF THE MODELS}

A typical example of the use of the models to represent the crystal structure of an amino acid or simple peptide is shown in Fig. 9, which is a photograph of a scale reproduction of one molecular layer in the crystal of $\mathrm{N}$-acetylglycine. The molecules are planar and are held together by $\mathrm{N}-\mathrm{H} \cdots \mathrm{O}$ and $\mathrm{O}-\mathrm{H} \cdots \mathrm{O}$ hydrogen bonds; the only other intermolecular contacts within the layer are between the hydrogen atoms of the $\mathrm{CH}_{2}$ and $\mathrm{CH}_{3}$ groups.

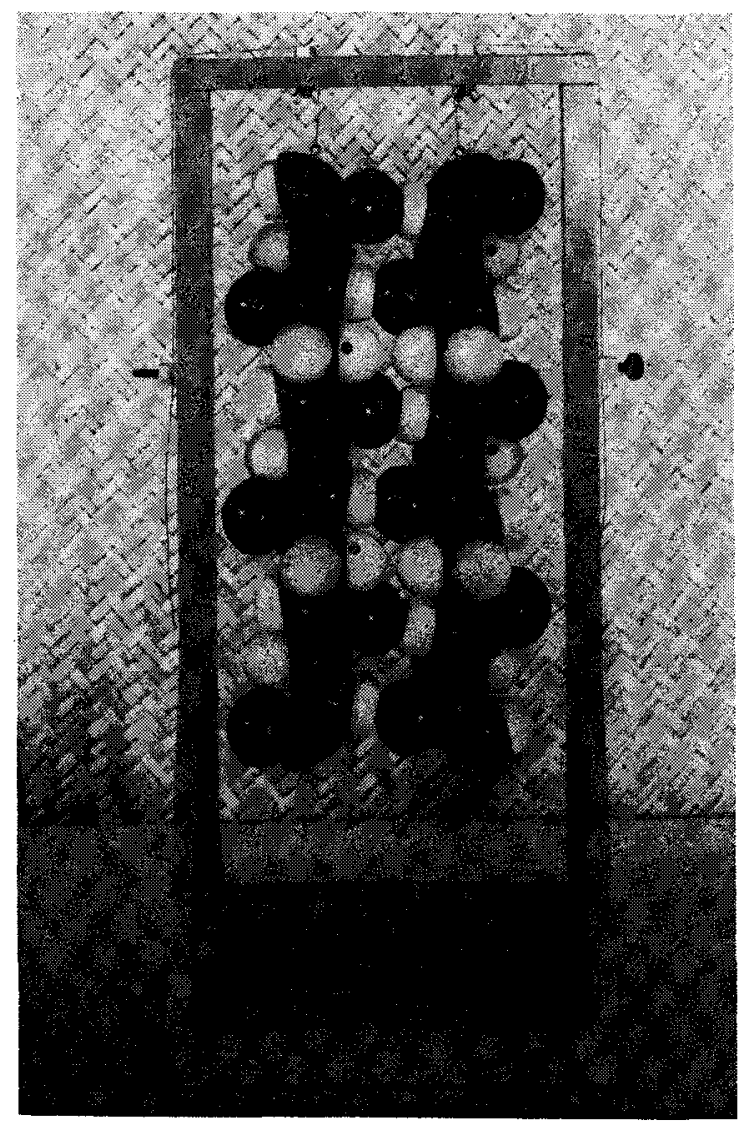

FIG. 11. A photograph of a suspension device for use in studying the packing of contiguous polypeptide chains.

${ }^{3} \mathrm{~J}$. Donohue and K. N. Trueblood, Acta Cryst. 5, 414, 419 (1952).
A model of the polypeptide chain in the form of the 3.7-residue helix is shown in Fig. 10. There is strong evidence that this configuration is present in many proteins and synthetic peptides having the $\alpha$-keratin structure. ${ }^{1}$ The helix shown in Fig. 10 represents a chain of polyglycine. In proteins containing amino acids other than glycine the side chains occupy one of the two hydrogen positions on the $\alpha$-carbon atom. For investigating the possible configurations of polypeptide chains and especially for studying the packing of chains in conformity with the x-ray diffraction data obtained from proteins, the device illustrated in Fig. 11 has been found to be extremely useful.

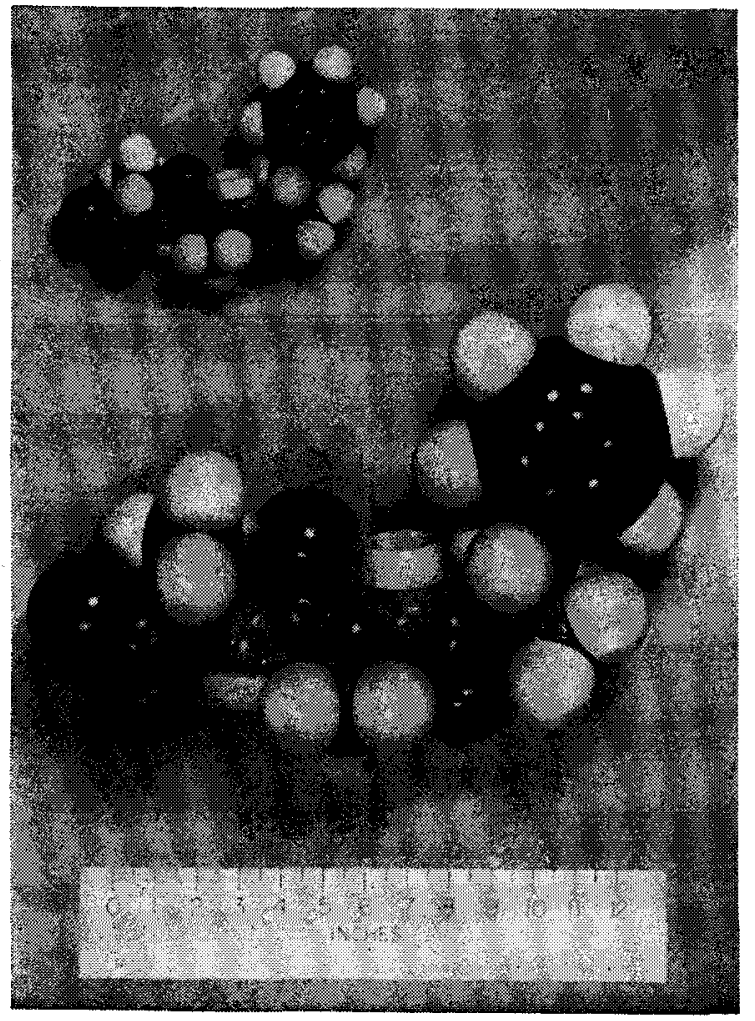

FIG. 12. A photograph comparing the dimensions of a halfscale plastic model of phenylalanylalanylalanine with those of a full-scale $(1 \mathrm{in} .=1 \mathrm{~A})$ wooden model.

\section{SMALL-SCALE PLASTIC MODELS}

Because of their weight and bulk, models built to the scale 1 in. $=1 \mathrm{~A}$ are inconvenient for work with large molecules or long polypeptide chains. We have therefore built a complementary set of models on half this scale. At first, they too were made of wood; because of their small size and different purpose they were fastened together by snap fasteners instead of internal clamps. These smaller models were indeed very useful. Their principal disadvantages were the high cost of manufacture and the lack of friction between bonded atoms which permitted them to rotate so easily around the bonds that particular molecular configurations were not ${ }^{*}$ 
well maintained. We have now greatly improved these models in both respects by casting them from colored, rubber-like vinyl plastic. The friction between the bonded faces reduces the freedom of rotation around the bonds, but permits the molecular configuration to be altered easily. The slight deformation which is possible with the plastic models makes them more adaptable to structures with bond angles or bond lengths differing slightly from the normal values. Advantage is taken of this deformability in the design of a simple hydrogenbond hydrogen atom in which this property of the plastic renders unnecessary the elaborate two-piece construction used in the wooden models.

These smaller-scale models are very useful for qualitative studies of molecular configuration and packing, but they cannot be used as a substitute for calculations. They are therefore complementary to the more precise large-scale models, and are not capable of doing the work for which the larger models were expressly designed.
Neither the large nor the small-scale models are commercially available. Models of the peptide phenylalanylalanylalanine constructed on the large and the small scale are contrasted in Fig. 12.

\section{ACKNOWLEDGMENTS}

The development of the molecular models described in this paper has been a collaborative effort in which many members of the staff of the California Institute of Technology have participated. We are especially grateful to Roger Hayward and William W. Schuelke for their ingenious solutions of many of the problems of design and construction, to Delmer D. Dill for his excellent workmanship, and to many members of the Institute faculty for their criticisms and suggestions.

For five years this work was aided by a grant from the National Foundation for Infantile Paralysis; more recently it has been aided by a grant from the National Institutes of Health, U. S. Public Health Service.

\title{
The Adaptation of the Norelco High-Angle Spectrometer to the Measurement of the X-Ray Diffraction of Fibers
}

\author{
Barbara IrVIN AND F. B. BREAZEALE \\ Research Department, American Enka Corporation, Enka, North Carolina
}

(Received October 13, 1952)

\begin{abstract}
The use of the North American Philips spectrometer for the radial and azimuthal scanning of the transmitted $x$-ray diffraction of rayon tire yarns involved a change in the slit system from convergent to divergent, design of a rotating sample holder, a reproducible sample preparation and positioning, and calibration and control checks which guarantee that the $x$-ray intensity remain constant to \pm 2 percent as automatically recorded by the rate meter. Typical data are presented and compared with data obtained photographically.
\end{abstract}

\section{INTRODUCTION}

$T$ HE study of fibered polycrystalline structures, which finds important application in the fields of metallurgy and textiles, is primarily concerned, for practical purposes, with the azimuthal and radial intensity distributions of the Debye-Scherrer diffraction rings. Our interest in highly stretched rayon tire yarns requires more than qualitative examination. Fibers for commercial application, although exhibiting great differences in technical properties of strength, breaking elongation and fatigue, are closely similar from the standpoint of their ultimate molecular arrangement (and thence their wide-angle $x$-ray pattern).

With the advent of the Norelco high-angle spectrometer, ${ }^{1}$ we realized an opportunity to obtain, rapidly and accurately, a large amount of intensity data for samples which exhibit relatively slight and often subtle differences in intensity distribution. This paper gives our experience in adapting the Norelco spectrometer to the measurement of the radial and azimuthal intensity

\footnotetext{
1 Manufactured by North American Philips Corporation, 750 South Fulton Street, Mt. Vernon, New York.
}

distribution of rayon tire yarns to a precision of \pm 2 percent recorded intensity.

\section{EXPERIMENTAL}

\section{A. Adaptation of the Spectrometer}

As delivered to us, the spectrometer was equipped with a convergent, focusing slit system to be used with reflectance samples. We did not change the position of these three slits in any way, but we did change the width of all three so that they subtend the same angle. No focusing is possible with such a divergent slit system and no great resolution is expected. In the case of cellulose the large amount of noncrystalline material present gives a natural line breadth which would appear to be the limiting factor rather than any instrumental resolution. We found that $\frac{1}{4}^{\circ}$ slits were most useful for our purposes.

Because we believe that we are primarily concerned with the distribution of the molecular axes about the fiber axis, we designed a sample holder which would position a card of rayon perpendicular to the $x$-ray beam, rotating with the Geiger tube as it traverses the 